\title{
Computer Simulation Is an Undervalued Tool for Genetic Analysis: A Historical View and Presentation of SHIMSHON - A Web-Based Genetic Simulation Package
}

\author{
David A. Greenberg \\ Division of Statistical Genetics, Department of Biostatistics, Mailman School of Public Health, and the Department \\ of Psychiatry, Columbia University Medical Center and the New York State Psychiatric Institute, New York, N.Y., USA
}

\section{Key Words}

Linkage analysis · Association analysis - Mode of inheritance - GWAS - Type 1 error - Affected sib pairs • LOD scores

\begin{abstract}
Computer simulation methods are under-used tools in genetic analysis because simulation approaches have been portrayed as inferior to analytic methods. Even when simulation is used, its advantages are not fully exploited. Here, I present SHIMSHON, our package of genetic simulation programs that have been developed, tested, used for research, and used to generated data for Genetic Analysis Workshops (GAW). These simulation programs, now web-accessible, can be used by anyone to answer questions about designing and analyzing genetic disease studies for locus identification. This work has three foci: (1) the historical context of SHIMSHON's development, suggesting why simulation has not been more widely used so far. (2) Advantages of simulation: computer simulation helps us to understand how genetic analysis methods work. It has advantages for understanding disease inheritance and methods for gene searches. Furthermore, simulation methods can be used to answer fundamental questions that either cannot be answered by analytical approaches or cannot even be defined until the problems are identified and studied, using simulation. (3) I argue
\end{abstract}

that, because simulation was not accepted, there was a failure to grasp the meaning of some simulation-based studies of linkage. This may have contributed to perceived weaknesses in linkage analysis; weaknesses that did not, in fact, exist.

Copyright $\odot 2011$ S. Karger AG, Basel

\section{Introduction}

This paper is, first and foremost, a long-delayed promotion of computer simulation methods in genetic analysis. Having been trained in theoretical methods in chemistry [1], in which computer simulation approaches were taken for granted, I was among the first to use computer simulation to answer questions about the behavior of genetic analysis methods [2]. However, for decades, the statistical genetics community, a discipline dominated by theoretical analytical approaches, has spurned simulation modeling as a practical tool for advancing the field, thus ignoring a whole class of methodology that can answer fundamental questions about genetic analysis. Although simulation has become widely used for tasks such as determining type I error and comparing methods, it has seldom been wielded as a tool of fundamental exploration in the way that analytical methods are used. Ignoring these other applications of simulation methods has deprived the

\section{KARGER \\ Fax +4161306 1234 \\ E-Mail karger@karger.ch}

www.karger.com
(C) 2011 S. Karger AG, Basel

$0001-5652 / 11 / 0724-0247 \$ 38.00 / 0$

Accessible online at:

www.karger.com/hhe
David A. Greenberg

Division of Statistical Genetics, Department of Biostatistics

Mailman School of Public Health, Columbia University Medical Center

722 W. 168th Street, New York, NY 10032 (USA)

Tel. +1 212342 0488, E-Mail dag@ shallot.cpmc.columbia.edu 
field of the widespread use of an important approach in the limited workshop of methods for finding disease loci. The SHIMSHON package of simulation programs is an attempt to redress that deprivation (see below).

Below, I discuss what I believe are the origins and consequences of under-using such a powerful investigative tool, of missed opportunities and of wrong directions. I then discuss our efforts to fill the gap by providing a welltested and (relatively) straightforward web-based mechanism that will allow anyone to generate data that can be used to answer complex genetic questions. I give several examples of how simulation techniques, in which we know the genetic truth behind the data's origins, can be used to provide surprising answers to basic problems in genetic analysis.

\section{How Can We Gain Understanding of Complex Systems?}

Simulation gives us a way to understand complex systems' workings and to develop an intuition about their behavior. When properly done, simulation, like any working model, can reveal details about the system that are hidden unless one sees it work. The approach of using computer simulation to gain understanding of complex systems is well established in chemistry, physics, and other areas of biology, but has tended to be shunned in statistical genetics. As I will discuss, the lack of such a comprehensive tool may have contributed to the current disfavor in which linkage analysis is held.

\section{Linkage Analysis Out of Favor}

Linkage analysis has proven the most successful method for proving that a strong genetic contribution to a disease exists and for reliably showing where in the genome those genes are. How, then, could such a powerful and useful technique as linkage analysis have become modus non grata, if not outright abused, just at a time when, thanks to the availability of a rich density of markers, the technique was poised to be able to maximize its power to detect loci? It was 'replaced' in the minds of many investigators and funding agencies, by an approach to finding disease genes - genome-wide association studies (GWAS) - that dealt with genes but not with inheritance, made careful phenotype evaluation almost impossible because of the sample sizes it demanded, was concerned with risk rather than cause or genetic mechanism (and typically found risk alleles with odds ratios on the order of only 1.2), had no power when faced with likely allelic heterogeneity, was subject to false positives due to relatively mild levels of population stratification, and could not necessarily distinguish between genes that were strong contributors to disease expression and those that may have only a tertiary influence on the disease.

Several contributing factors led to the denigration of linkage and the widespread adoption of association analysis, including GWAS's requirement for only relatively simple and obvious data collection (i.e. independent cases and independent controls), the ease of understanding $2 \times 2 \chi$-squared tables (even if there were $10^{6}$ of them), the appeal of not having to collect family data, the appeal of using the newest technology in the easiest possible way, fashionability, and - according to some I have spoken with - not having to be too concerned with phenotype ('It's the law of large numbers.'). However, I believe that one of the most important factors working against linkage was psychological; the how and why of linkage analysis, its inner workings, was perceived as difficult to understand. Even the concept of identity by descent allele sharing, not to speak of LOD scores, can cause difficulties for students and for those who do not regularly consider these concepts (e.g. recombination fraction, linkage equilibrium, sharing probability, mating type frequency, etc.).

For example, anyone can devise an example of a relevant $2 \times 2$ table and demonstrate the effect of sample size or the number of controls on an association analysis calculation. Not so with linkage analysis. As a result, even experts, as we will see, can misunderstand the effects of assumptions and parameters on the outcome of linkage analyses. Many investigators (even theoreticians) do not have the expertise to answer some questions via analytical approaches (e.g. 'What is the correct null hypothesis when one is testing for a two-locus model?'). This leaves them no option but to accept what others tell them. However, computer simulation can easily allow one to answers questions both mundane and complex if the software is available.

\section{Simulation and the Perceived Importance of the Inheritance Model in Linkage Analysis}

\section{Linkage and Segregation Analysis}

In 1989, I published a paper entitled, 'Determining Mode of Inheritance by Maximization of LOD Scores'. At that time in the history of human genetic analysis, virtually the only two methods available were segregation analysis and linkage analysis. Each had its drawbacks. 
Although the tools were available, the ability to actually carry out a linkage analysis was limited. The problem was that there were only about 30 known markers available. (Note for younger readers: yes, that means 30 markers for the entire genome!) RFLPs (restriction fragment length polymorphisms) were only recently available, but would soon be followed by microsatellites, which proved more convenient and informative than RFLPs and would give linkage analysis a wide reach. Thus, linkage explorations of the entire genome were becoming possible and linkage's successes with Mendelian diseases demonstrated its power to find disease-causing loci. There were high hopes for linkage's application to common diseases.

However, at the time, a major issue confronting linkage analysis was how to cope with the fact that, for diseases with complex inheritance, the mode of inheritance is unknown, but a mode of inheritance had to be assumed in order to do a linkage analysis. The major concern was that assuming the wrong mode of inheritance could lead to false positives and false negatives. The dogma was that one had to do a segregation analysis before one did a linkage analysis so that one could assume the 'correct' mode of inheritance.

Moreover, there were problems with segregation analysis. With a history dating back to Gregor Mendel, segregation analysis tests whether an assumed mode of inheritance (dominant, recessive, polygenic, or any other possible genetic mechanism) can be statistically rejected in a collection of family data, usually identified through affected family members. The critical word, however, is 'rejected' - the method could not 'prove' a mode of inheritance. Also, for anything but the simplest models, the number of families required could be high [3].

However, a critical problem with segregation analysis had to do with ascertainment bias [4-7]. To give a very simple example of the problem: families are ascertained through affected family members. However, some mating types are 'at risk' but produce no affected offspring (and therefore cannot be ascertained). If investigators do not take this into account, their analysis can be badly distorted. Moreover, this failure to include in the analysis mating types with no affected offspring is only one of many factors that can distort a segregation analysis. Dealing with these factors entails choosing an ascertainment model, which, except in extreme cases, is elusive. In fact, for most studies, it is virtually impossible to know the true ascertainment model, a finding that we showed using simulation [4] and that was later shown theoretically [5] (albeit from a different viewpoint).

A Historical View and Presentation of SHIMSHON
Parameter Estimation by Maximizing LOD Scores

The work I published in 1989 [8] was motivated by these problems. The paper showed, by generating realistic data via simulation and then analyzing those data, that one could reliably determine the mode of inheritance at a locus by maximizing the maximum LOD score with respect to the model (parameters), and that the mode of inheritance determination was, as far as my explorations went, not affected by ascertainment (but see [9]). Furthermore, not only was it possible to get a reliable estimate of the penetrance by LOD score maximization, but the actual value of the maximum LOD score was relatively robust to the penetrance assumption, meaning that if the assumed penetrance was inexact, it made little difference in linkage detection.

These results were contrary to what was accepted in the genetic analysis community, so I asked several colleagues for opinions, including Dr. Robert Elston. He confirmed that my findings went against the accepted theory, which said that the mode of inheritance could not be estimated by maximizing max LOD scores. Nonetheless, I submitted the work to the American Journal of Medical Genetics. The then-editor, Dr. John Opitz, accepted the paper and, because the reviews were controversial, asked Dr. Elston to write an editorial commenting on the approach and results.

\section{The Case against Computer Simulation: Man Bites \\ Dog}

What happened in Dr. Elston's editorial represents an interesting episode in the sociology of statistical genetic analysis. Just a few months after Dr. Elston had said that he could see no theoretical justification for these results, in his editorial [10] he proved that theory did support the simulation results (because the distribution of the LOD score under the null hypothesis is independent of the mode of inheritance assumption). However, the fact that the editorial backed up the simulation results, while an important fact, is not what makes the story interesting.

His editorial's major thrust was that the theoretical insight supporting the simulation result demonstrated that simulation studies were of no use for uncovering underlying principles. That is why the editorial was entitled, 'Man Bites Dog?' because my paper proposed an 'absurd' reversal of the natural order (i.e. simulation showing something that analytical methods cannot). However, in a critical omission, there is no word in the editorial about how either the simulation or the analytical findings affect the practice of doing linkage analysis. One can now only speculate, but the lack of discussion of the results' impli-

Hum Hered 2011;72:247-257 
cations for finding disease loci may have had an important negative effect on the further development of linkage analysis. Had there been acknowledgement that LOD score maximization was an effective way to deal with the mode of inheritance problem, the false promises and misunderstandings of affected-only methods, and especially sib pair analysis methods [11] might have been avoided. Maximization of LOD scores, the method that has the most power to detect important loci and simultaneously yield information about those loci (e.g. mode of inheritance), might have been more widely used. (What happened instead we discuss below.)

Thus, the editorial may not only have reinforced the prevalent negative view of simulation studies, but may also have profoundly affected how linkage analysis was actually carried out in analyses of real data.

As noted, at that time, the question of the mode of the inheritance assumption was viewed as the critical problem in linkage analysis. What the 1989 paper showed by simulation, and what Dr. Elston proved analytically, was that the mode of inheritance could be estimated consistently. Therefore, the mode of inheritance assumption did not represent a major impediment to linkage analysis. We went on to confirm and expand that conclusion in subsequent papers (e.g. [12-14]) by showing that approximations to the true mode of inheritance were all that was needed in order to reliably detect disease loci, even when the modes of inheritance were complex. Yet, what became generally accepted was that the less powerful strategy of collecting only affected sib pairs was optimal for linkage because no mode of inheritance assumption was necessary to do the analysis.

\section{The Sickly Ears of Corn Devour the Healthy Ones (Genesis, 41:7)}

Starting about 1990, affected sib pair (ASP) methods were quickly embraced because: (a) unlike complex linkage calculations, ASP analysis could, almost literally, be done on the back of an envelope, and (b) there was no need to assume a mode of inheritance. This led to the belief that ASP methods (and later other affected-only analyses) somehow transcended the true underlying mode of inheritance. The idea that, suddenly, the true mode of inheritance no longer mattered for the analysis came about in part because those capable of showing analytically (as opposed to by simulation) that this was nonsense did not immediately engage the problem. It was not until 1994 that Knapp et al. [15] showed that the commonly-used 'mean test' for ASP linkage has a rejection area identical to that of a LOD score analysis assuming recessive inheritance. Thus, the actual mode of inheritance of the disease did matter and ASP methods lost significant power if the underlying mode of inheritance was dominant [16]. Later Whittemore [17] showed that for any method designed to use only affected family members (and make no parameter assumptions, so-called 'non-parametric' methods), there is a LOD score analysis with some mode of inheritance assumption that provides the same statistical characteristics as the affected-only method. In other words, an 'affecteds-only' analysis is the statistical equivalent of assuming some mode of inheritance; one just does not know what it is, cannot change it, and cannot even determine if it is plausible. However, by that time, collecting sib pairs for ASP analysis had become the accepted data collection scheme for hunting common disease loci.

The strategy of collecting the absolute minimum amount of phenotypic information in families (i.e. only the affected members) not only limits the available families but leads to ignoring other available informationcontaining data. These data include not only supposedly unaffected family members but also the data that can come from careful phenotypic analysis of all family members. Collecting such phenotypic information often reveals subclinical markers and those data are critical in finding disease-related loci in common disease.

It is possible that, had the implications of either the original simulation finding about mode of inheritance and/or Dr. Elston's proof been understood, richer data sets (whole families with clinical information), rather than ASP data sets, might have become the norm. But ASP-based approaches also have the advantage that they are analytically tractable and understandable. This tractability led to the publication of any number of analytical elaborations of an underpowered approach [16] that could also lead to further misinterpretation [18].

Had simulation methods been generally available and more widely accepted, testing of the propositions then abounding could have been done by anyone in a position to use them. There is, of course, always the question of using the available tools appropriately.

\section{LOD Score Maximization Not Considered}

Here is an example of the issues that arose concerning simulation, mode of inheritance, ASP versus LOD scores, and the non-acceptance of LOD score maximization as a 
viable approach. Dizier et al. [19] tested whether ASP or LOD score analysis had more power to detect linkage in a complex model. They simulated data for a number of complex epistatic two-locus disease models and then ran a segregation analysis on the simulated data. They used the segregation-derived parameters for LOD score linkage analyses (which, as explained, was once considered the 'correct' way to perform a linkage analysis) and compared the results to ASP analyses. The LOD score analysis with the segregation-derived parameters gave stronger evidence for linkage than did ASP analysis in all but three models; for those three models, the ASP analysis yielded higher power. The authors concluded that there are circumstances under which ASP analysis is superior to LOD scores. These results were later cited [20] as a reason not to use LOD scores.

However, Durner et al. [21] showed that these conclusions were misleading. By generating data under the same inheritance models used by Dizier et al., Durner et al. showed that LOD scores maximized with respect to mode of inheritance had more power for all the generating models than either ASP analysis or the LOD score analyses incorporating segregation analysis-derived parameters; they also showed why that was the case and that maximizing LOD scores had more power to detect linkage than other affecteds-only ('non-parametric') methods.

Why Dizier et al. used only segregation analysis-derived parameters in their analysis, rather than also maximizing LOD scores with respect to mode of inheritance, is puzzling but may be related to the tone in 'Man Bites Dog,' which could have unintentionally tarred the finding with the same brush that tarred the method that led to it.

\section{The Advantages and Limitations of Simulation}

An obvious use of simulation is the testing of new genetic analysis approaches. By using simulated data, one knows the true mechanism and parameters from which the data come and, therefore, one can better assess how close the analysis program comes to the true values. Even more importantly, one can see what happens when the assumptions of the analysis do not, in fact, match the genetic basis used to generate the data. This would seem to be an obvious, if not required, aspect of publishing any new analysis method or program. However, for years the standard has been to, first, test that the method returns values that match the theoretically predicted ones when the inputs do match the method's assumptions, and then to test the program using actual disease data for which the

A Historical View and Presentation of SHIMSHON true parameters are unknown. Then, whatever one finds can be declared evidence that the method works. As a result, there are analysis programs available to use for which one has no idea how they will behave if the assumptions of the method are violated. This lacuna may be, in part, due to lack of easy access to data that can be simulated using a large variety of models. This is a lack we have sought to remedy (see below).

The presumed limitations of simulation studies expressed in Dr. Elston's editorial were shared - and continued to be shared - by many statistically-trained investigators in genetic analysis. In this view, simulation should be 'limited' to those questions that cannot be solved analytically, or should be used only to investigate robustness, or in cases where realistic sample sizes are too small to approach asymptotic behavior. Simulations created and tailored only for the more mundane tasks may not apply beyond the narrow questions for which they were designed. For example, the assumed distribution(s) may be too restricted or may be based on assumptions that apply only to large samples, or critical parameters (such as $\alpha$-levels) may be fixed. Even worse, the simulations may have been designed to produce only data that match the assumptions of the methods being tested and may not be capable of generating data that violate those assumptions.

Problems that could be solved analytically are often not solved because, as in the case with maximization of LOD scores, the answer was believed to be already known or because, as the examples below show, no one thought of the question.

\section{Simulation and Analytical Approaches Look at the World in Different Ways}

Like simulation, analytic methods make assumptions that can restrict the generality of the underlying models in order to make the calculations possible. Thus, the argument that simulation methods are 'particular' is true, but it is often true of analytic methods, as well. With a robust and well-tested simulation program available, the restrictions can easily be diminished to the point of nonexistence, while eliminating similar restrictions for analytical methods may pose major problems (e.g. see example 1, below). Moreover, analytical methods are frequently called upon to explain, or model, mathematically what is known to be true from observation. Simulation, on the other hand, can be the source of those observations in the first place. 
For example, the Greenberg 1989 paper used a uniformly distributed random variable to describe the probability of a subject being affected given that they had the disease genotype, i.e. random reduced penetrance. However, if a trait is caused by two epistatically-interacting loci, the 'penetrance' at either locus is no longer random but is correlated among family members. Thus, the socalled penetrance is reduced if one analyzes data assuming a single locus model. (The penetrance for the disease genotype (the genotypes at both loci taken together) is 1.0.) We later posed the following question: if one performs a LOD score analysis assuming the existence of only one of the two loci but with reduced penetrance, how does this affect one's ability to find either locus? To try and answer this question analytically may be difficult, but answering it by simulation, as we did for two-point $[14,22,23]$ and for multipoint analysis [13], was relatively simple, straightforward, and comprehensive.

\section{Other Uses for Simulation Studies: Three Examples}

In the example of the Dizier et al. work (above), we saw that limiting the exploration of the problem, and not asking enough 'what if...' questions, may represent the biggest problem in simulation's application. Simulation's power lies in its exploratory ability and its ability to help us understand the size and shape of the problem.

I offer three examples of how simulation has been used to raise and then answer fundamental questions about how genetic analysis methods work.

\section{Example 1: Dependence of Type 1 Error Distributions} on Sample Size in Multipoint Linkage Analysis

A typical example of simulation's use in answering questions can be seen in [24]. This paper arose as a direct response to a mostly analytically-based paper by Xing and Elston [25] on type I error in multipoint linkage analysis.

By means of extensive computer simulations we were able to acquire a broad picture of how type I error behaves for multipoint analysis when those analyses are done precisely as one would in carrying out analyses on real data. We determined that, under the null hypothesis of no linkage, and for a fixed cutoff value, the actual number of times that multipoint linkage analyses indicate significant evidence for linkage decreases with sample size. Thus, for a given cutoff value, the type I error probability goes down as the number of families in a data set increases (fig. 1a). Furthermore, we determined that this is true for both dominant and recessive modes of inheritance, at all levels of penetrance, and even when the LOD score is maximized with respect to mode of inheritance (mod scores) (fig. 1b).

This was a startling and yet encouraging discovery. It has at least three practical consequences for interpreting multipoint linkage results: (1) If, for any pre-selected LOD score cutoff, the actual probability of a false positive is a function of sample size, then the chosen value for a cutoff for significant evidence for linkage is not only variable, but could be notably lower than the nominal value of 3.0 that has been accepted for decades. For better or worse, the finding suggests that indications of linkage should not be simply ignored because they do not reach pre-determined significance levels; e.g. a multipoint LOD score of 1.0 might, under the right conditions, be statistically significant evidence of linkage. Potentially, this vastly expands the power of multipoint linkage to detect loci. (2) The sample size dependence comes about because the genomic region under actual examination is restricted. (The analog under two-point analysis is if only recombination fractions between, say, 0.0 and 0.2 were considered. In that case, also, type I error decreases with sample size (see [24]).) This last point we showed analytically, again demonstrating that a good working model will deepen our understanding by showing us phenomena that we can then prove and explain using analytical tools. (3) The ancient controversy of what should be the significance cutoff for LOD scores is rendered moot. One cannot answer the question without a consideration of multiple factors.

Thus, by using simulation as an exploratory tool, one can gain new insights into methodology more easily than by using analytical methods alone.

\section{Example 2: Imprinting Detection from Linkage Data}

A major advantage of simulation is that one can easily ask what happens when the assumptions of any particular method are violated. Even more far afield from the usual approaches to understanding methods, simulation gives us the ability to take advantage of 'anomalies' in methods that can give us tools to see problems from different angles. That is the case with detecting genetic imprinting by comparing independent male-female recombination fractions.

Imprinting is usually (and correctly) viewed as a differential penetrance that depends on an allele's parental origin in the mother or father. In an analysis of epilepsy data [26], we stumbled on another way of detecting imprinting: as a differential in the apparently sex-dependent 

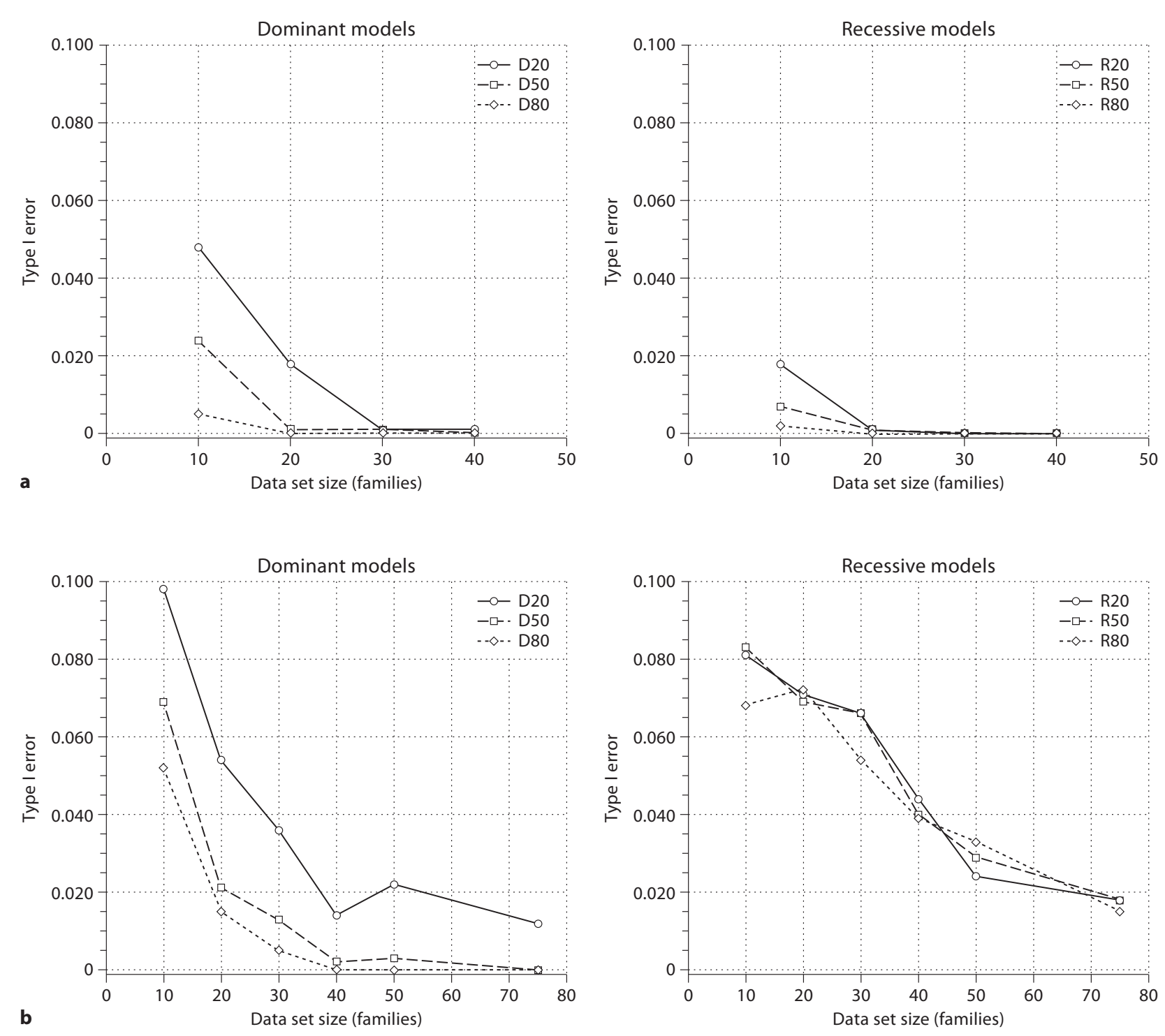

Fig. 1. Type I error for LODs maximized over $10 \mathrm{cM}$. a LODs. The actual type I error probability goes down - in both dominant and recessive modes of inheritance, at all levels of penetrance - as the number of families in a data set increases. b MODs. This is true even when the LOD score is maximized with respect to mode of inheritance (mod scores). Taken from [24] (with permission).

recombination fraction (an approach that had been suggested by Smalley [27]). I say 'apparently' because in truth there is no difference in the recombination fraction between the sexes in the genomic area we were examining. Rather, we observed that, consistently in our families in the epilepsy dataset, linkage evidence always came from the mother, never from the father.

The only explanation, besides a true male-female difference in the recombination fraction in the region, was that there had to be imprinting. The only way to test the hypothesis was by using simulation, because there appeared to be no readily available analytical approach to determining if this observation was merely an anomaly.

In the resulting work [28], we used simulation to explore both the direct method (modeling parent-specific penetrance) and the indirect approach of using differential recombination fraction. We found both methods had strengths and weaknesses. The most surprising finding 


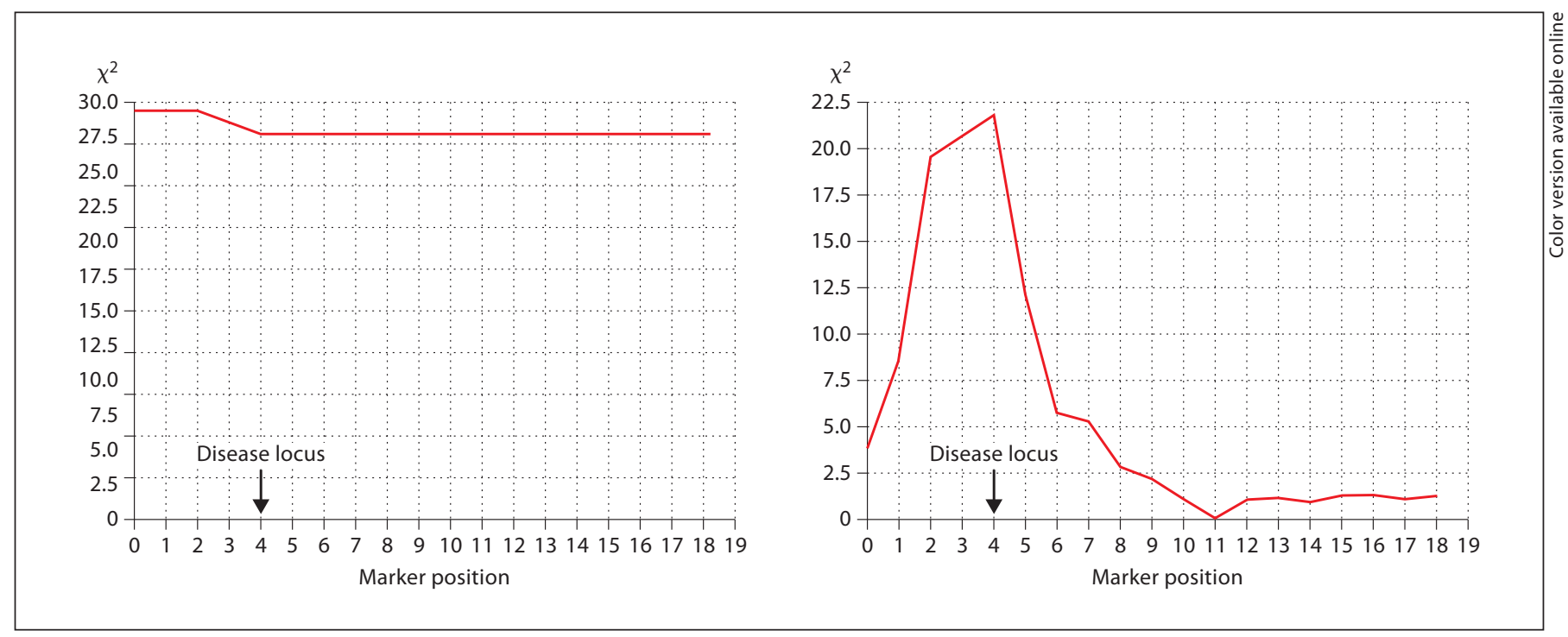

Fig. 2. Left panel: case-control analyses across 18 marker loci with $D^{\prime}=1.0$ between all loci and the identical allele frequency (0.5) at each marker. Right panel: case-control analyses across 18 marker loci with D' $=1.0$ and random allele frequencies at the 18 markers.

was that certain confounders that reduced evidence for linkage could actually enhance evidence for imprinting. We found that while genetic heterogeneity reduced the power of penetrance-based modeling for detecting imprinting, it actually increased the power to detect imprinting via the differential recombination fraction approach, while in the parent-specific penetrance modeling, a reduction in penetrance appeared to improve imprinting detection.

\section{Example 3: Haplotype Blocks?}

This example will illustrate how useful the ability to simulate and analyze data can be to understand what to expect from an analysis.

It has become widely accepted that so-called 'haplotype blocks' exist throughout the genome. These blocks consist of regions of stable DNA in which there have been few recombinations over time within a population. Because of their stability and the high linkage disequilibrium (LD) between the markers, typing one or a few SNPs in the region would then tell us all we need to know about which haplotype is being inherited. In theory, this would allow one to identify a disease-containing haplotype without typing many SNPs on the same piece of DNA.

With our simulation program, one can ask: given no recombination and given complete LD along a stretch of
DNA, what would the evidence for association look like for SNPs in the block? One might expect an equivalent amount of association evidence for each of those SNPs along that stretch of DNA, and that is exactly what one sees (fig. 2a, left panel). In this example, the disease allele frequency is 0.01 , dominantly inherited with $50 \%$ penetrance. The markers all had a gene frequency of 0.5 . LD (D') was 1 between all loci.

We now ask, what is the effect of variable gene frequency of the markers on this block? Taking advantage of the program's ability to set the marker frequencies randomly, we set marker frequencies between 0.1 and 0.9 randomly for each marker. The result is shown in figure 2 , right panel. We see here that the 'block' no longer exists. The evidence for association peaks where expected, but quickly falls as one moves away from the disease gene location. The conclusion is that one must be wary of what 'blocks' can tell us and that one must choose one's markers carefully.

\section{Web-Available Simulation Tools: The SHIMSHON Package}

We have worked over the past 20 years to develop a suite of genetic simulation programs that are as general as we could make them. We have constantly used them, 
upgraded them, created specialized versions (e.g. to answer the imprinting question), and corrected bugs.

Correction of 'errors' is no small problem when advocating using simulation in the same way one uses analytical techniques. What we have observed, with what can only be described as frightening frequency, is that the results of simulation can contradict 'common sense', or even the accepted theory on which one bases one's tests of the simulator. Above, I have described three examples of this: (1) the observation that maximizing the LOD score is a valid and consistent way to estimate genetic parameters; (2) the finding that the type I error rate in multipoint analysis is a function of sample size; (3) the observation that, when imprinting exists, factors that lower the evidence for linkage can increase the evidence for imprinting. Each of these observations set off intense rounds of testing: performing close examination of the family structures, marker and disease segregation, and confirming that proportions of affected members, etc., were in accordance with the input parameters. In the first two examples discussed above, the finding was ultimately supported by analytical approaches and in the last, by comparing methods using two approaches that had nothing in common.

Earlier versions of these programs were used to generate data for genetic analysis workshops (GAW) 11 [29] and 14 [30]. The GAW 11 data not only amounted to a stringent test of the programs but provided new ideas on how to improve the programs, ideas that were used later for the simulation of the GAW 14 data. The creativity brought to the analyses of the data that year again illustrates how simulation can be used as a research tool [31]. While the main purpose of the GAW 11 simulation was to explore complex genetic models, the GAW 14 simulation added the problem of complex phenotype definition, with the simulated data designed with the perceived phenotype complexity of the real alcoholism data offered for analysis [32]. (The LD as implemented in data for GAW 14 (a last minute add-on at the time) is greatly improved in the current program.)

In an attempt to give investigators access to reliable, tested, simulation machinery, we have made this package web-accessible and user-friendly and, given the complexity of the problem, usable. (I do not say 'easy', because the only thing 'easy' about genetic analysis is the ease of making mistakes.) The programs in the package are also ideally suited for teaching, and we have used them that way for a number of years.

The web-based system is available at http://potato. cpmc.columbia.edu:8087/shimshon.jsp. It consists of three different simulation and analysis packages that allow the user to set a large number of parameters to characterize the simulated data. The system also allows one to analyze the data in a number of ways by permitting one to specify analysis parameters (mode of inheritance(s), penetrance(s), sporadic rate, marker allele frequencies, disease allele frequencies, number of markers (for multipoint), marker map, etc.). One can also designate two loci to be the disease loci. One can then determine the nature of the interaction between the loci (epistasis, heterogeneity, or many configurations in between). In addition, one can control the ascertainment criteria: type of family simulated (e.g. nuclear family, three-generation pedigrees, etc.) and families of specific size or configuration (e.g. affected sib pairs, at least 2 affected offspring in generation 3 , family sizes of at least 5 , etc.).

The three different programs embody three different levels of complexity and realism. These are: (1) 'Shimshon', (2) 'Jezebel', and (3) 'Caleb'. In all cases, the parameters used to analyze the data can be different from those used to generate the data.

A defining aspect of the programs is that they can produce data using two disease loci. The parameters can be set so that there is interaction between the loci, or they can each produce the same trait independently, or almost any relationship in between. One can also model sporadics (non-inherited disease).

Below, we describe each program. We have connected different analysis programs to the simulations so that analyses can take place automatically after the simulations are done including GENEHUNTER [33], ALLEGRO [34], or MERLIN [35], although not all three are available on the web-based version yet. (We have extensively tested all three and, with rare exceptions, determine that all three perform equally well for most analyses of the simulated data.) Alternatively, the user can download the simulated raw family data, the program input files, etc. for any analyses one wishes to do locally.

Shimshon generates family data for two-point linkage analysis, i.e. one disease and one marker locus. However, a second disease locus can be set either for epistasis or heterogeneity experiments. This second locus is not linked to either the marker or to the first disease locus. Although two-point linkage analysis is now seldom used in actual studies, it excels as a teaching tool for understanding the basics of human genetics and the concepts involved in quantitative genetic analysis (e.g. recombination, LOD scores, heterogeneity, etc.). A single-locus model is effected by setting the disease allele frequency at the second locus to zero. Sporadics are effected by setting the gene 
frequency at the second locus to 1.0 and setting the penetrance at the second locus to the desired sporadic rate.

Jezebel simulates multipoint linkage data. The version currently in use simulates a 'chromosome' of up to 20 loci, two of which are disease loci. (As with the two-point version of the program (Shimshon), to simulate a one-locus disease model, the disease allele frequency at one of the disease loci is set to zero.) The twenty loci can be placed at any genetic distance from each other, and the gene frequencies at the markers can be set either to user-determined values or to random values between pre-set limits. The number of alleles at the markers can be set to between two and nine. Again, the disease loci can be set to act independently (heterogeneity) or epistatically (twolocus disease model), or the nine-element penetrance matrix can be used to create a wide variety of different kinds of inheritance models, including additive and intermediate models. The marker map used to analyze the data can be set independently of the true (generating) map, so that one can understand the effect of location misspecification (a problem that has not entirely disappeared from actual data). Jezebel does not simulate LD.

Caleb also simulates multipoint linkage data. It allows the user to analyze the data for linkage but additionally allows the user to specify LD between the loci and to perform association analyses, both case-control and familybased. LD is also specified independently of recombination fraction, so that one can specify high LD and high recombination fraction, e.g. to simulate an admixed population. In addition to being able to invoke linkage analysis, the user may also specify case-control association analysis and/or family-based (TDT (transmission disequilibrium test)-type) association analysis, which allows one to compare the methods. It helps understand both the differences and similarities of linkage and association. Caleb can give the user a fundamental perspective not only on linkage and association, but also an understanding of the strengths and weaknesses of genetic analysis in general. It was used to generate data for one of the first explorations of population stratification on case-control association studies [36]. It still works by generating whole populations and then selecting subjects/families from them. This approach gives the program enormous flexibility.

\section{The Philosophical Viewpoint}

We need discipline-wide mechanisms in genetic analysis, such as the military uses, to look into what works and what does not. The military is devoted to ob- serving what happened in a battle, noting what went wrong and what went right, and learning from the mistakes and successes. There seems to be no such mechanism in the current state of genetic analysis. We seem to lurch from new data collection paradigms to new analysis methods without taking stock of why the last one did not work out as we expected. For example, accepting as truth, and spending resources on, the notion of 'common disease, common variant' and, when that proves inadequate, change the second 'common' in the phrase to 'rare' and claiming that now we have discovered the truth and should spend our resources there, is priming for more disillusionment.

I would like to make it harder for someone to make an unchallengeable pronouncement about any aspect of genetic analysis (such as was said about mode of inheritance and linkage analysis) and have it treated as a pre-accepted fact simply because there are only five people in the world with the expertise to answer the question analytically. We have now put into reach of anyone a system - or the start of a system - that will permit scientists to simulate interesting real-world situations and then test the effects of those situations on possible conclusions. These are situations that will arise in formulating genetic studies and that involve mode of inheritance, ascertainment scheme, heterogeneity, etc. Some may be horrified at the thought of allowing the semi-numerate the ability to challenge sophisticated theory, but such reversals often come with new technologies. The edged weapons-fighting expertise of medieval knights took years of training, but was ultimately no match for untrained peasants with firearms. I fully acknowledge the possibility of abuse, but the possibility of abuse would be better than accepting as truth pronouncements that cannot be tested or are difficult to test, as has happened in the past. Besides, when the tools are available to everyone, abuse will be easier to detect and counter. We need the ability to test questions at a practical or heuristic level. That is part of the challenge of constructing any wide-ranging system.

\section{Acknowledgements}

This work was supported in part by grants NS27941, NS061829, MH48858, and NS070323. Thanks to Drs. Susan Hodge and William Stewart for criticizing the manuscript more than once. 


\section{References}

1 Greenberg DA, Barry CD, Marshall GR: Investigation and parametrization of a molecular dielectric function. J Am Chem Soc 1978;100:4020-4026.

$>2$ Greenberg DA: Simulation studies of segregation analysis: application to two-locus models. Am J Hum Genet 1984;36:167-176.

$>3$ MacLean CJ, Morton NE, Lew R: Analysis of family resemblance. IV. Operational characteristics of segregation analysis. Am J Hum Genet 1975;27:365-384.

4 Greenberg DA: The effect of proband designation on segregation analysis. Am J Hum Genet 1986;39:329-339.

5 Vieland VJ, Hodge SE: Inherent intractability of the ascertainment problem for pedigree data: a general likelihood framework. Am J Hum Genet 1995;56:33-43.

$\checkmark 6$ Hodge SE, Vieland VJ: The essence of single ascertainment. Genetics 1996;144:12151223.

$>7$ Morton NE: Genetic tests under incomplete ascertainment. Am J Hum Genet 1959;11:116.

$>8$ Greenberg DA: Inferring mode of inheritance by comparison of lod scores. Am J Hum Genet 1989;34:480-486.

$>9$ Vieland VJ, Hodge SE: The problem of ascertainment for linkage analysis. Am J Hum Genet 1996;58:1072-1084.

$>10$ Elston RC: Man bites dog. The validity of maximizing lod scores to determine mode of inheritance. Am J Hum Genet 1989;34:487488.

$>11$ Hodge SE, Elston RC: Lods, wrods, and mods: the interpretation of lod scores calculated under different models. Genet Epidemiol 1994;11:329-342.

12 Greenberg DA, Abreu P, Hodge SE: The power to detect linkage in complex disease by means of simple lod-score analyses. Am J Hum Genet 1998;63:870-879.

13 Greenberg DA, Abreu PC: Determining trait locus position from multipoint analysis: accuracy and power of three different statistics. Genet Epidemiol 2001;21:299-314.

14 Greenberg DA: Linkage analysis assuming a single-locus mode of inheritance for traits determined by two loci: inferring mode of inheritance and estimating penetrance. Genet Epidemiol 1990;7:467-479.
15 Knapp M, Seuchter SA, Baur MP: Linkage analysis in nuclear families. 2. Relationship between affected sib-pair tests and lod score analysis. Hum Hered 1994;44:44-51.

16 Hodge SE: Exact ELODS and exact power for affected sib pairs analyzed for linkage under simple right and wrong models. Am J Med Genet 1998;81:66-72.

17 Whittemore AS: Genome scanning for linkage: an overview. Am J Hum Genet 1996;59: 704-716.

18 Huang J, Vieland VJ: Comparison of 'modelfree' and 'model-based' linkage statistics in the presence of locus heterogeneity: single data set and multiple data set applications. Hum Hered 2001;51:217-225.

19 Dizier MH, Babron MC, Clerget-Darpoux F: Conclusions of lod-score analysis for family data generated under two-locus models. Am J Hum Genet 1996;58:1338-1346.

20 Kidd KK: Can we find genes for schizophrenia? Am J Med Genet 1997;74:104-111.

21 Durner M, Vieland VJ, Greenberg DA: Further evidence for the increased power of lod scores compared with nonparametric methods. Am J Hum Genet 1999;64:281-289.

22 Vieland VJ, Hodge SE, Greenberg DA: Adequacy of single-locus approximations for linkage analyses of oligogenic traits. Genet Epidemiol 1992;9:45-59.

23 Vieland VJ, Greenberg DA, Hodge SE: Adequacy of single-locus approximations for linkage analyses of oligogenic traits: extension to multigenerational pedigree structures. Hum Hered 1993;43:329-336.

24 Hodge SE, Rodriguez-Murillo L, Strug LJ, Greenberg DA: Multipoint lods provide reliable linkage evidence despite unknown limiting distribution: type I error probabilities decrease with sample size for multipoint lods and mods. Genet Epidemiol 2008;32:800-815.

25 Xing C, Elston RC: Distribution and magnitude of type I error of model-based multipoint lod scores: implications for multipoint mod scores. Genet Epidemiol 2006;30:447-458.

26 Greenberg DA, Durner M, Keddache M, Shinnar S, Resor SR, Moshe SL, Rosenbaum D, Cohen J, Harden C, Kang H, Wallace S, Luciano D, Ballaban-Gil K, Tomasini L, Zhou G, Klotz I, Dicker E: Reproducibility and complications in gene searches: linkage on chromosome 6 , heterogeneity, association and maternal inheritance in juvenile myoclonic epilepsy. Am J Hum Genet 2000; 66:508-516.
27 Smalley SL: Sex-specific recombination frequencies: a consequence of imprinting? Am J Hum Genet 1993;52:210-212.

28 Greenberg DA, Monti MC, Feenstra B, Zhang J, Hodge SE: The essence of linkagebased imprinting detection: comparing power, type 1 error, and the effects of confounders in two different analysis approaches. Ann Hum Genet 2010;74:248262.

29 Greenberg DA, MacCluer JW, Spence MA, Falk CT, Hodge SE: Simulated data for a complex genetic trait (problem 2 for GAW11): how the model was developed, and why. Genet Epidemiol 1999;17:S449S459.

30 Greenberg DA, Zhang J, Shmulewitz D, Strug LJ, Zimmerman R, Singh V, Marathe $S$ : Construction of the model for the genetic analysis workshop 14 simulated data: genotype-phenotype relationships, gene interaction, linkage, association, disequilibrium, and ascertainment effects for a complex phenotype. BMC Genet 2005; 6(suppl 1):S3.

31 Greenberg DA: Summary of analyses of problem 2 simulated data for GAW11. Genet Epidemiol 1999;17(suppl 1):S429-S447.

32 Vieland VJ: Heterogeneity: GAW group 15. Genet Epidemiol 2005;29(suppl 1):S110S115.

33 Kruglyak L, Daly MJ, Reeve-Daly MP, Lander ES: Parametric and nonparametric linkage analysis: a unified multipoint approach. Am J Hum Genet 1996;58:1347-1363.

34 Gudbjartsson DF, Thorvaldsson T, Kong A, Gunnarsson G, Ingolfsdottir A: Allegro version 2. Nat Genet 2005;37:1015-1016.

35 Abecasis GR, Cherny SS, Cookson WO, Cardon LR: Merlin - rapid analysis of dense genetic maps using sparse gene flow trees. Nat Genet 2002;30:97-101.

36 Heiman GA, Hodge SE, Gorroochurn P, Zhang J, Greenberg DA: Effect of population stratification on case-control association studies. I. Elevation in false positive rates and comparison to confounding risk ratios (a simulation study). Hum Hered 2004;58:3039. 